\title{
Latest results from the Pierre Auger Observatory
}

\author{
Isabelle Lhenry-Yvon ${ }^{1, a}$ for the Pierre Auger Collaboration ${ }^{2, b}$ \\ ${ }^{1}$ Institut de Physique Nucléaire d'Orsay (IPNO), Université Paris 11, CNRS-IN2P3, Orsay, France \\ ${ }^{2}$ Observatorio Pierre Auger, Av. San Martiín Norte, 304, 5613 Malargüe, Argentina
}

\begin{abstract}
The Pierre Auger Observatory has been designed to investigate the origin and nature of Ultra High Energy Cosmic Rays (UHECR) with energies from $10^{17}$ to $10^{20} \mathrm{eV}$. In this paper we will review some of the most recent results obtained from data of the Pierre Auger Observatory, namely the spectrum of cosmic rays, the anisotropies in arrival directions and the studies related to mass composition and to the number of muons measured at the ground. We will also discuss the implication of these results for assembling a consistent description of the composition, origin and propagation of cosmic rays.
\end{abstract}

\section{Introduction}

The origin of the UHECR (above $10^{18} \mathrm{eV}$ ) is still a challenge today. Even though cosmic rays have been discovered more than 100 years ago, their study remains of great interest, especially at the highest energies where their existence is still a puzzle for astrophysicists. At these energies, they can only be observed indirectly through air showers initiated in the Earth's atmosphere. The Pierre Auger Observatory [1], conceived to characterize the properties of the UHECRs, is a hybrid instrument for air shower detection. It combines an array of particle detectors, the Surface Detector array (SD) [2], to sample the air shower front as it reaches the ground, and Fluorescence Detector (FD) telescopes to collect the ultraviolet light emitted by nitrogen as showers develop in the atmosphere [3]. The SD consists of 1660 water-Cherenkov stations, spread over a total area of $\approx 3000 \mathrm{~km}^{2}$ on a $1.5 \mathrm{~km}$ grid spaced close to Malargüe, province of Mendoza, Argentina; it measures the lateral density distribution of charged particles at the ground and their temporal distribution in each station. The FD overlooks this region from four peripheral sites, each with six FD telescopes and records shower longitudinal profiles. A small area of $\sim 30 \mathrm{~km}^{2}$, the Infill, is instrumented with 71 surface detectors on a $750 \mathrm{~m}$ grid and with three FD with elevated fields of view to lower the energy threshold of the Observatory down to $10^{17} \mathrm{eV}$. A set of high quality instruments is installed in the field to monitor the atmospheric conditions; on-line and long-term performances of the detectors and data quality are monitored continuously [4]. The overall uptime of the SD is above $98 \%$ and it is $100 \%$ efficient in detection showers above $3 \times 10^{18} \mathrm{eV}$, while the FD, which can operate only in clear moonless nights, is operational only $13 \%$ of the time. Thanks to the possibility of combining the information from the two types of detectors, the reconstruction capabilities are enhanced with respect to the individual detectors.

\footnotetext{
a e-mail: 1henry@ipno.in2p3.fr

${ }^{\mathrm{b}}$ Full author-list: http://www.auger.org/archive/authors_2014_09.html
} 

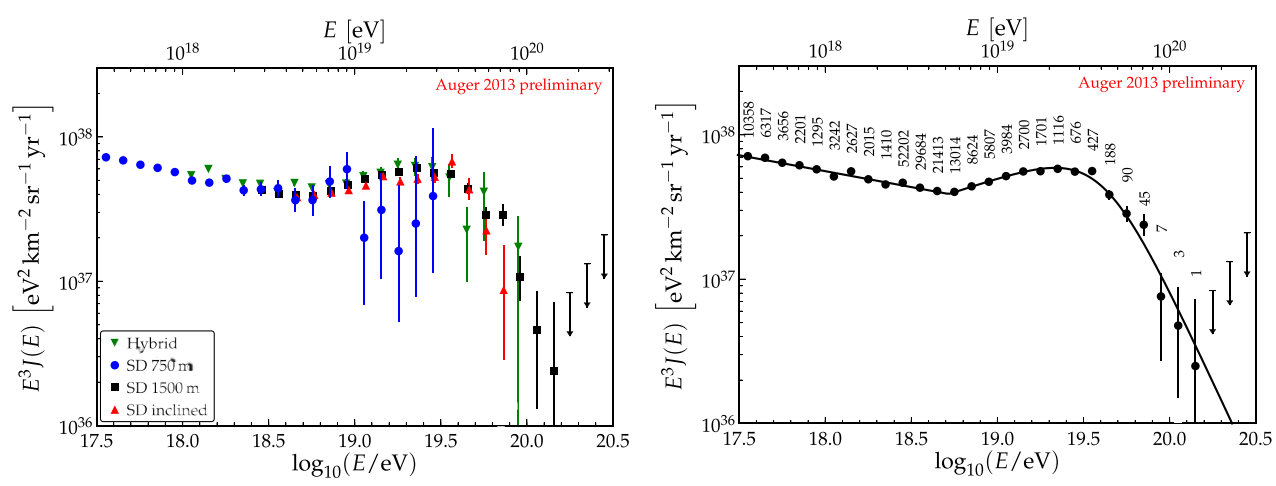

Figure 1. Left: the energy spectra obtained from hybrid events, and from data of the $750 \mathrm{~m}$ and $1500 \mathrm{~m}$ SD arrays for vertical and inclined showers measured at the Auger Observatory. Right: the combined spectrum, with the number of events shown in each energy bin. The arrows represent upper limits at an $84 \%$ confidence level [5].

\section{Spectrum}

Four different data sets are used to compose the Pierre Auger Observatory spectrum: events with the $750 \mathrm{~m}$ SD subarray and events with the $1500 \mathrm{~m}$ full SD array, for events with zenith angle below $55^{\circ}$ and $60^{\circ}$ respectively (hereafter referred as vertical events), hybrid events measured with both the FD and the SD, events with zenith angle above $60^{\circ}$ (called inclined events). Those spectra are shown in Fig. 1, left. The flux $J(E)$ is multiplied by $E^{3}$ to favor an easy identification of features in the power-law spectrum $E^{-\gamma}$.

These data sets are combined to compose the spectrum shown in Fig. 1, right, using a maximum-likelihood method providing the relative normalization. Smearing corrections are applied to take into account possible bin-to-bin migrations. About 130,000 events compose this spectrum. The systematic uncertainty in the energy scale is 14\% [6] and the energy resolution for events measured only with the SD is better than $12 \%$ above $10^{19} \mathrm{eV} \mathrm{[5].}$ Two features can be clearly identified in the resulting spectrum: a sharp hardening of the spectrum at the so-called ankle, and a suppression of the flux at the highest energies. At the energy of $5 \times 10^{18} \mathrm{eV}$, corresponding to the ankle, the power-law index changes from $3.23 \pm 0.07$ to $2.63 \pm 0.04$. Above $2 \times 10^{19} \mathrm{eV}$ the spectrum starts to deviate from this power law, being suppressed with respect to what would be expected by extrapolating the spectrum above the ankle. At $4 \times 10^{19} \mathrm{eV}$ the flux is half of that expected from that extrapolation. The significance of the suppression is $20 \sigma$.

Details of the Auger spectrum were compared with theoretical predictions assuming different extragalactic astrophysical scenarios, pure-light or pure-heavy compositions, and two maximal acceleration energies at the sources, $10^{20}$ and $10^{20.5} \mathrm{eV}$. Auger flux measurements over the whole energy range from below the ankle up to the suppression region are in better agreement with predictions assuming a maximal acceleration energy of $10^{20} \mathrm{eV}$, and exclude a pure-iron composition. The energy spectrum of UHECRs turns out to be most likely the result of multiple processes involving the spatial distribution of their sources, the acceleration process and the interactions along their propagation paths. To distinguish between the different scenarios mentioned above and thus to understand the origin of the highest energy cosmic rays, complementary measurements of the mass composition are necessary as well as studies of the anisotropy in the arrival directions. 

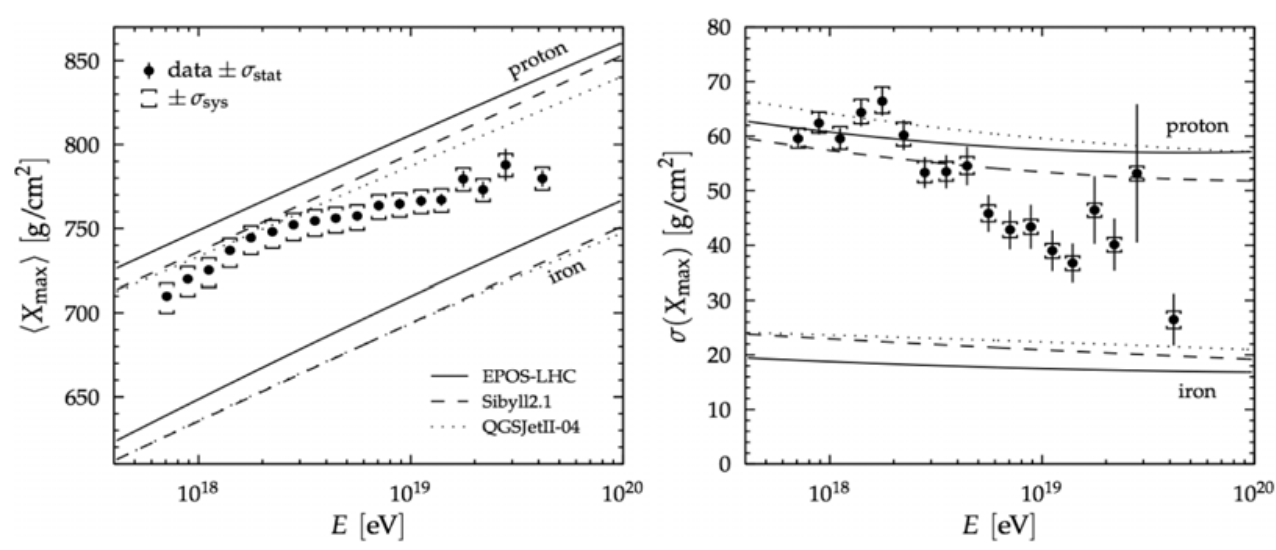

Figure 2. $\left\langle X_{\max }\right\rangle$ and its RMS as a function of energy, compared to the predictions of the most recent, post-LHC, air shower simulations using different hadronic interaction models (see text) [10].

\section{Composition studies}

The Pierre Auger Observatory hybrid detectors measure air shower observables containing information about the mass of the primary particle. The fluorescence telescopes allow us to follow the longitudinal development of the electromagnetic component of the air showers in the atmosphere and thus to measure the depth of maximum development, $\left\langle X_{\max }\right\rangle$, and its fluctuations, $\sigma\left(X_{\max }\right)$. A difference of $\sim 100 \mathrm{~g} / \mathrm{cm}^{2}$ is expected between the $\left\langle X_{\max }\right\rangle$ of showers generated by protons and iron nuclei, the latter developing higher in the atmosphere. A narrower distribution (smaller $\sigma\left(\mathrm{X}_{\max }\right)$ ) is also expected from heavy nuclei as compared to light ones. $\left\langle X_{\max }\right\rangle$ is related to the depth of the first interaction of the primary and to the subsequent development of the shower; for this reason, the interpretation in terms of composition is complicated by the large uncertainties in the hadronic interaction models used in the simulations.

Once corrected for the detector resolution, $\left\langle X_{\max }\right\rangle$ and $\sigma\left(\mathrm{X}_{\max }\right)$ can be directly compared to the predictions of the latest models, Sybill 2.1 [7], QGSJetII-04 [8] and EPOS-LHC [9]. As shown in Fig. 2, all models predict a constant elongation rate as a function of energy for pure compositions. This is not compatible with our measurements, which clearly show a change of slope at a few EeV. If we assume that no change in hadronic interactions comes into play at these energies, our data show evidence for a composition getting heavier above the ankle region.

The analysis of $\left\langle X_{\max }\right\rangle$ and $\sigma\left(\mathrm{X}_{\max }\right)$ relies on measurements of hybrid events, thus suffering from the lower number of events measured during the shorter duty cycle of the FD.

The nature of the primaries can be studied with more statistics by exploiting the SD data. The time structure of particles reaching the surface detector array on the ground allows us to measure the distribution of muon production distances and from that distribution infer the slant depth at which the production of muons reaches its maximum, $\left\langle X_{\max }^{\mu}\right\rangle$. The depths of both maxima are, of course, strongly correlated, mainly through the depth of the first interaction. At the same time, these measurements provide a means of testing and constraining models of hadronic interactions at the energies involved in the showers. The analysis of $\left\langle X_{\max }^{\mu}\right\rangle$ exploits the higher number of showers measured with the SD, and therefore also reaches higher energies. Results from simulations indicate that this quantity depends on the mass of the primary particle in a similar way to $\left\langle X_{\max }\right\rangle$, its average and dispersion being 

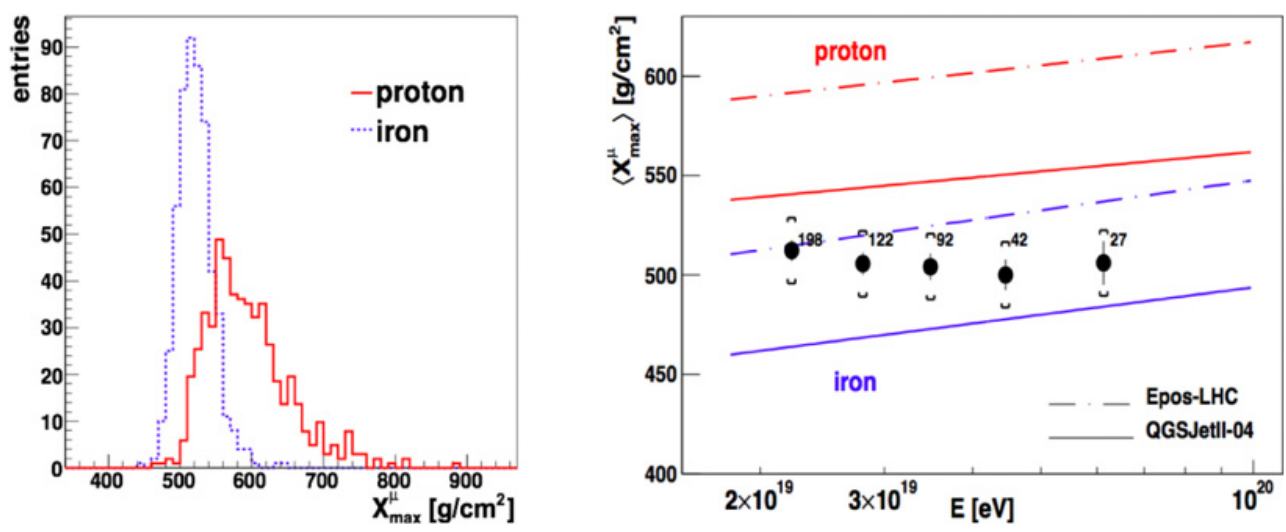

Figure 3. Left: $\left\langle X_{\max }^{\mu}\right\rangle$ distributions in showers initiated by protons and iron nuclei of $3 \times 10^{19} \mathrm{eV}$ and angles between $55^{\circ}$ and $65^{\circ}$, simulated with EPOS-LHC. Right: evolution of $\left\langle X_{\max }^{\mu}\right\rangle$ measured versus energy, compared to the predictions of simulations using EPOS-LHC and QGSJetII-04 for proton and iron showers. The brackets represent the systematic uncertainty. The number of showers is shown for each energy bin [11].

smaller for heavier primaries compared to those obtained for lighter ones. An example of the $\left\langle X_{\max }^{\mu}\right\rangle$ distribution for showers initiated by protons and iron nuclei of $3 \times 10^{19} \mathrm{eV}$ is shown in Fig. 3, left, obtained with simulations using EPOS-LHC as the interaction model.

The analysis of muon production depths was performed on showers of energies above $2 \times 10^{19} \mathrm{eV}$ detected at the Pierre Auger Observatory from January 2004 up to December 2013. In the construction of the distributions of muon production depths only muons from inclined showers with zenith angles between $55^{\circ}$ and $65^{\circ}$ and within a distance from the shower core between $1700 \mathrm{~m}$ and $4000 \mathrm{~m}$ were included, thus allowing a clear selection of the signal due to the muonic component, without any bias arising from the electromagnetic component. In Fig. 3, right, the resulting $\left\langle X_{\max }^{\mu}\right\rangle$ are shown for five energy bins, and compared to predictions from simulations adopting QGSJetII-04 or EPOS-LHC. Both models predict a linear evolution of $\left\langle X_{\max }^{\mu}\right\rangle$ with the logarithm of the energy, although with a systematic difference of about $50 \mathrm{~g} / \mathrm{cm}^{2}$. The data points are bracketed by the lines following predictions of the simulations for proton and iron primaries with the QGSJetII-04 model, and fall below those predicted from simulations with EPOS-LHC. Furthermore, the data show a flatter trend than predicted for a pure-proton or a pure-iron composition. From these results it is also possible to use the muon production depth as a tool to constrain hadronic interaction models.

In the frame of the same interaction models, both $\left\langle X_{\max }^{\mu}\right\rangle$ and $\left\langle X_{\max }\right\rangle$ can be converted to $\langle\ln \mathrm{A}\rangle$, the mean logarithmic mass, hopefully converging to a coherent result [11]. As shown in Fig. 4, there is a discrepancy in the EPOS-LHC case, which could be most probably explained as a collective effect of nuclei in the atmosphere (as compared to the proton-proton interactions at LHC) [5].

As for QGSJetII-04, the good agreement is only apparent, as this model does not accurately reproduce the rapidity gap distribution of LHC data. From these considerations, although insufficient to exclude specific models, it appears that the UHECR data have the power to constrain high energy interaction models in unexplored energies and kinematic regions. 

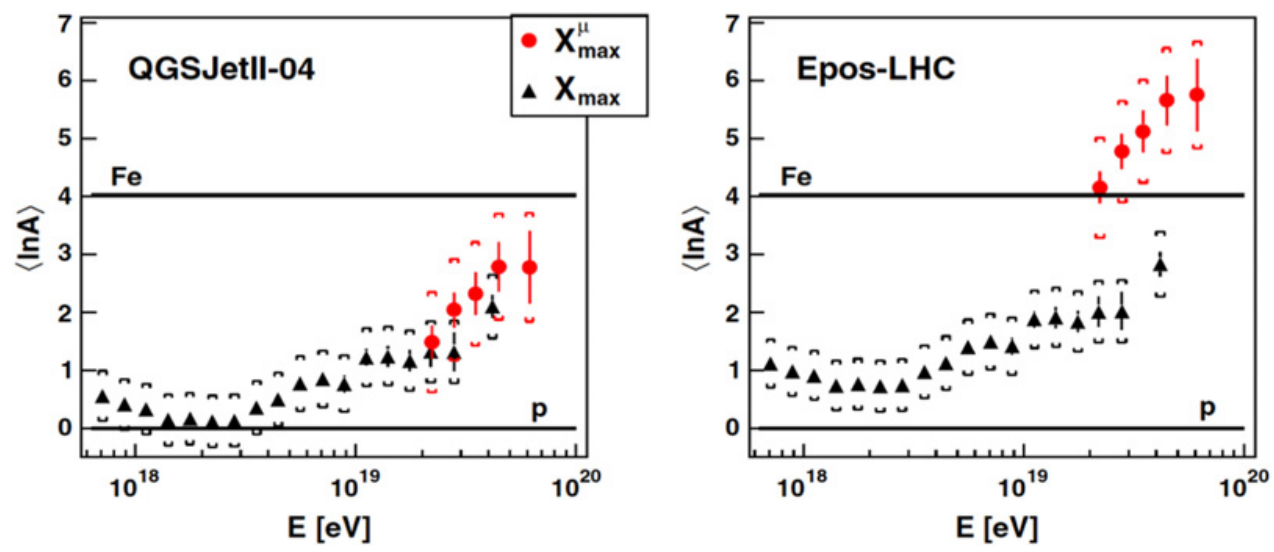

Figure 4. Conversion of $\left\langle X_{\max }\right\rangle$ (black triangles) and $\left\langle X_{\max }^{\mu}\right\rangle$ (red circles) to $\langle\ln \mathrm{A}\rangle$, as a function of energy, for the hadronic interaction models QGSJetII-04 (left) and EPOS-LHC (right).

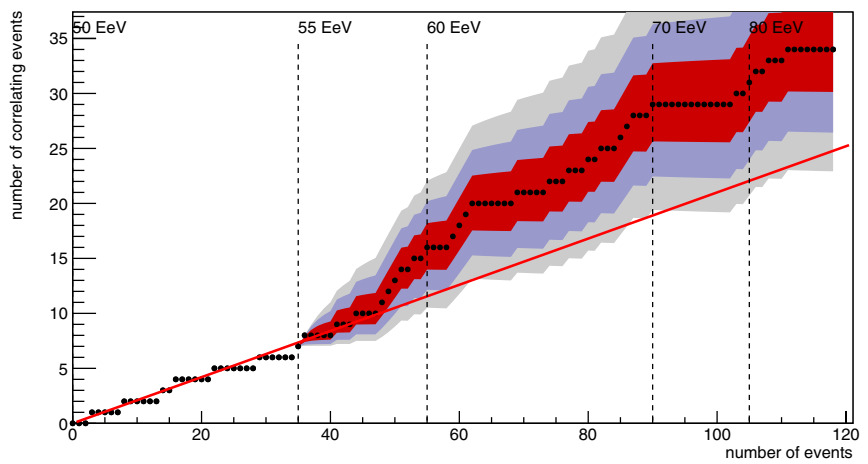

Figure 5. Auger events correlating with the positions of AGN from the VCV catalogue, in an energy ordered plot [5].

\section{Anisotropies in arrival directions}

A key observable to obtain information about the sources and nature of UHE cosmic rays is the distribution of their arrival directions. At these energies, the particles are most probably extragalactic; if the observed flux suppression can be attributed to propagation effects, they would be limited by a GZK horizon of about $100 \mathrm{Mpc}$. A correlation between the arrival directions of the highest energy cosmic rays (above $50 \mathrm{EeV}$ ) and the positions of the nearby AGN from the Véron-Cetty-Véron (VCV) catalogue was reported in 2007 [12]. The most recent update of this search is shown in Fig. 5 where the current estimate of the fraction of correlation is $(33 \pm 5) \%$, against a $21 \%$ expected under the hypothesis of isotropy.

The observation of large scale anisotropies in the arrival directions could give a hint on the galactic or extragalactic origin of the cosmic rays in different energy ranges. Recently, the Pierre Auger Collaboration and the Telescope Array Collaboration published the results for the dipole and quadrupole moments, combining their data sets of arrival directions above $\sim 10^{19} \dot{\mathrm{e} V}$ in a full-sky coverage. The region of the sky for which the fields of view of both observatories overlap provides the cross-calibration factor for the different exposures and the compensation of systematic uncertainties due to different energy scales. No significant deviation from isotropy at any scale is found throughout the various analyses. Upper limits 
on the amplitude of the dipole and quadrupole moments are derived as a function of the direction in the sky, resulting between $7 \%$ and $13 \%$ for the dipole, and between $7 \%$ and $10 \%$ for a symmetric quadrupole [13].

\section{Conclusion}

Results from the Pierre Auger Observatory clearly confirmed the called ankle transition in the spectrum index at $4 \times 10^{18} \mathrm{eV}$ as well as the flux suppression above $5 \times 10^{19} \mathrm{eV}$. These results are not enough to understand whether the suppression is caused by the energy loss of cosmic rays during their propagation, or is rather a signal of the maximum acceleration at their sources. The measurement of the composition of the detected cosmic rays is a crucial key to answer this question and to understand their origin. For this reason the Pierre Auger Collaboration is currently proposing an upgrade of the Observatory, that consists in an addition of scintillator detectors aiming at a better identification of the chemical composition of the primary particles, mostly increasing the mass sensitivity of the Observatory in the highest-energy region. It will also allow an improved study of anisotropies by selecting those primaries with a small charge and of hadronic interactions at center-of-mass energies an order of magnitude above those presently attainable at the LHC.

\section{References}

[1] J. Abraham et al. (Pierre Auger Collaboration) Nucl. Instrum. Meth. A 523, 50-95 (2004)

[2] J. Abraham et al. (Pierre Auger Collaboration) Nucl. Instrum. Meth. A 613, 29-39 (2010)

[3] J. Abraham et al. (Pierre Auger Collaboration) Nucl. Instrum. Meth. A 620, 227-251 (2010)

[4] P. Abreu et al. (The Pierre Auger Collaboration) J. Instrum. 7, P09001 (2012)

[5] A. Letessier-Selvon for the Pierre Auger Collaboration, Proc. 33rd ICRC, arXiv:1310.4620 (2013), Braz. J. Phys. 44, 560-570 (2014)

[6] V. Verzi for the Pierre Auger Collaboration, Proc. 33rd ICRC, arXiv:1307.5059 (2013)

[7] E.-J. Ahn et al., Phys. Rev. D 80, 09400 (2009)

[8] S. Ostapchenko, Phys. Rev. D 83, 014018 (2011)

[9] T. Pierog et al., arXiv:1306.0121 [hep-ph](2013)

[10] A. Aab et al. (The Pierre Auger Collaboration) Phys. Rev. D 90, 122005 (2014)

[11] A. Aab et al. (The Pierre Auger Collaboration) Phys. Rev. D 90, 012012 (2014)

[12] The Pierre Auger Collaboration, Science 318, 938 (2007)

[13] A. Aab et al. (The Pierre Auger and Telescope Array Collaborations) Astrophys. J. 794, 172 (2014) 